“C 2016 IEEE. Personal use of this material is permitted. Permission from IEEE must be obtained for all other uses, in any current or future media, including reprinting/republishing this material for advertising or promotional purposes, creating new collective works, for resale or redistribution to servers or lists, or reuse of any copyrighted component of this work in other works." 


\title{
Hybrid Tree-rule Firewall for High Speed Data Transmission
}

\author{
Thawatchai Chomsiri, Xiangjian He*, Priyadarsi Nanda, Zhiyuan Tan
}

\begin{abstract}
Traditional firewalls employ listed rules in both configuration and process phases to regulate network traffic. However, configuring a firewall with listed rules may create rule conflicts, and slows down the firewall. To overcome this problem, we have proposed a Tree-rule firewall in our previous study. Although the Tree-rule firewall guarantees no conflicts within its rule set and operates faster than traditional firewalls, keeping track of the state of network connections using hashing functions incurs extra computational overhead. In order to reduce this overhead, we propose a hybrid Tree-rule firewall in this paper. This hybrid scheme takes advantages of both Tree-rule firewalls and traditional listed-rule firewalls. The GUls of our Tree-rule firewalls are utilized to provide a means for users to create conflict-free firewall rules, which are organized in a tree structure and called 'tree rules'. These tree rules are later converted into listed rules that share the merit of being conflict-free. Finally, in decision making, the listed rules are used to verify against packet header information. The rules which have matched with most packets are moved up to the top positions by the core firewall. The mechanism applied in this hybrid scheme can significantly improve the functional speed of a firewall.
\end{abstract}

Index Terms-Firewall, High Speed Firewall, Network Security, Computer Network, Cloud Network

\section{INTRODUCTION}

$\mathrm{F}_{\mathrm{s}}$ rewalls were first invented in 1990s [1], and have been developed to operate more securely and faster. Since the first generation firewalls, the commercially used firewalls still perform network traffic regulation based on listed rules. The listed rules are a set of rule sequences which consist of conditions and actions. If information carried in the header fields (e.g., Source IP, Destination IP and Destination Port) of an incoming packet is matched with the condition of a rule, the packet will be accepted or denied in accordance with the action specified in the rule. However, in the listed-rule set of a traditional firewall, there may be 'shadowed rules' [2] and/or redundant rules. On one hand, shadowed rules may cause security problems because protection rules could be shadowed by other rules listed ahead. On the other hand, redundant rules cause latency in traffic processing and lower the throughput of a network due to the undesirable waste of time on verifying against these rules. The detailed discussion of these problems can be found in our previous work published in [3].

To address the afore-mentioned problems, we recently proposed a new type of firewall called 'Tree-rule firewall' in [4]. It has been proved that the Tree-rule firewall guarantees no conflicts (e.g., no shadowed rules and no redundant rules) in rule sets, and is more efficient in traffic

- Thawatchai Chomsiri is with the University of Technology Sydney (UTS), PO Box 123, Broadway 2007, Australia. E-mail: Tha-

watchai.Chomsiri@student.uts.edu.au.

- ${ }^{*}$ Corresponding author. Xiangjian He is with the University of Technology Sydney (UTS), PO Box 123, Broadway 2007, Australia. E-mail: Xiangjian.He@uts.edu.au.

- Priyadarsi Nanda is with the University of Technology Sydney (UTS), PO Box 123, Broadway 2007, Australia. E-mail: Priyadarsi.Nanda@uts.edu.au.

- Zhiyuan Tan is with the University of Twente, P.O. Box 217 7500AE Enschede, Netherlands.E-mail: z.tan@utwente.nl. processing in comparison with traditional listed-rule firewalls [4]. In our recent follow-up study [5], a new stateful mechanism was proposed to further improve the Tree-rule firewall with the capability of tracking the states of network connections. In comparison with IPTABLES, the most popular open source firewall, the stateful Treerule firewall is more advanced in terms of processing speed.

However, complex hashing computations are involved in the stateful mechanisms used in the Tree-rule firewall and the IPTABLES. A hashing function has to be invoked at least once in either the stateful Tree-rule firewall or the IPTABLES in stateful mode to verify each single packet travelling through the firewall. It takes approximately 1,400 nanoseconds to compute the Jenkins hash (jhash) [6] used in these two firewalls running on a standard PC with a Pentium 2.4 GHz CPU. Whereas, comparing two variables takes only 1.4 nanoseconds with the same setup. On contrary, if an incoming packet matches with the first rule in a stateless firewall (e.g., IPTABLES in stateless mode), then the firewall needs to conduct comparisons between four packet header fields (i.e., Source IP address, Destination IP address, Source Port and Destination Port) and the respective conditions specified in the rule. This rule matching is approximately $1400 /\left(1.4^{*} 4\right)=250$ times faster than that of a stateful firewall.

Although the traditional stateless firewalls (e.g., IPTABLES in stateless mode) can operate fast, the rule conflict problem is still the main obstacle for improving firewall speed using the rule sequence tuning. In a firewall rule list, there may be many frequently matched rules which are positioned at the bottom of the list. These rules, especially the last rule which was created to deny all packets, cannot be moved up to the top positions because rule conflicts may cause the change of firewall policy if 
TABLE 1

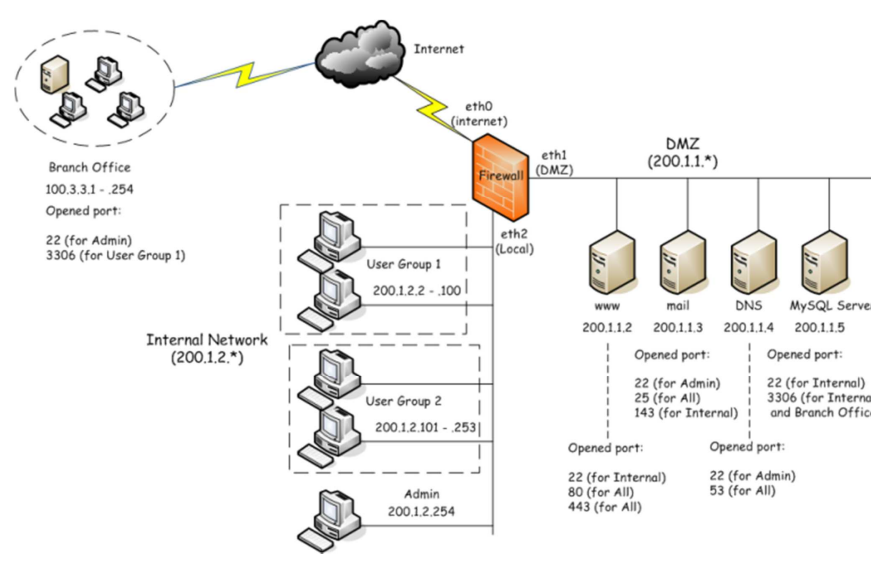

Fig. 1. An example network.

they are moved up. However, if frequently matched rules in a firewall can be moved up to top positions, the firewall, especially a firewall working in a large network with a huge number of rules, will operate faster.

Motivated by the above, the contributions of this paper are shown as follows.

- We propose a hybrid firewall which takes advantages of both the Tree-rule and stateless mechanism in design. This scheme ensures no rule conflicts and high traffic processing speed in nature. More frequently matched rule will be moved to higher positions in the rule list automatically.

- We derive a mathematical model measuring the time consumption in the hybrid firewall and a mathematical model for measuring the efficiency of data transmission. The experimental results show a great improvement in terms of efficiency on the proposed firewall.

- The proposed firewall is implemented under a cloud environment. The experimental results show that the proposed hybrid firewall using 'automatic rule sorting' outperform the ones with 'non-automatic rule sorting' modes.

The rest of this paper is organized as follows. The background and the related work are introduced in Section 2. Our proposed hybrid firewall scheme is then detailed in Section 3. The implementation of our proposed scheme is presented and the experimentation is demonstrated in Section 4. Finally, conclusion is drawn along with the discussion of our future research in Section 5.

\section{BACKgROUNd AND RELATEd WORK}

Previous research approaches aiming to enhance functional speed of firewalls can be categorized into three types. The first type focuses on discovery and elimination of rule conflicts, especially redundant rules, to reduce the rule size of a firewall. This can reduce memory space consumption and processing time on a firewall. The second type emphasizes on developing firewalls with high performance hardware, such as implementing a firewall on
A SET OF LISTED RULES CREATED FOR AN EXAMPLE NETWORK IN

FIG. 1.

\begin{tabular}{|c|c|c|c|c|}
\hline $\begin{array}{l}\text { No: } \\
===:\end{array}$ & $\begin{array}{l}\text { Source_IP } \\
==========\end{array}$ & $\begin{array}{l}\text { Dest_IP } \\
=======\end{array}$ & $\begin{array}{l}\text { Dest_Port } \\
=========\end{array}$ & Action \\
\hline 1 & $100.3 .3 . *$ & 200.1 .1 .5 & 3306 & Accept \\
\hline 2 & $100.3 .3 . *$ & 200.1 .1 .2 & 80 & Accept \\
\hline 3 & $100.3 .3 . *$ & 200.1 .1 .2 & 443 & Accept \\
\hline 4 & $100.3 .3 . *$ & 200.1 .1 .3 & 25 & Accept \\
\hline 5 & $100.3 .3 . *$ & 200.1 .1 .4 & 53 & Accept \\
\hline 6 & $100.3 .3 . *$ & * & * & Deny \\
\hline 7 & 200.1 .1 .* & $100.3 .3 . *$ & * & Deny \\
\hline 8 & $200.1 .1 . *$ & $200.1 .2 . *$ & * & Deny \\
\hline 9 & $200.1 .1 .^{*}$ & * & * & Accept \\
\hline 10 & $200 \cdot 1 \cdot 2 \cdot 2-100$ & $100.3 .3 . *$ & 3306 & Accept \\
\hline 11 & 200.1 .2 .254 & $100.3 .3 . *$ & 22 & Accept \\
\hline 12 & $200.1 .2 . *$ & $100.3 .3 . *$ & $\star$ & Deny \\
\hline 13 & $200.1 .2 . *$ & 200.1 .1 .2 & 22 & Accept \\
\hline 14 & $200.1 .2 .{ }^{*}$ & 200.1 .1 .2 & 80 & Accept \\
\hline 15 & $200.1 .2 . *$ & 200.1 .1 .2 & 443 & Accept \\
\hline 16 & 200.1 .2 .254 & 200.1 .1 .3 & 22 & Accept \\
\hline 17 & 200.1 .2 . $^{*}$ & 200.1 .1 .3 & 25 & Accept \\
\hline 18 & $200.1 .2 . *$ & 200.1 .1 .3 & 143 & Accept \\
\hline 19 & 200.1 .2 .254 & 200.1 .1 .4 & 22 & Accept \\
\hline 20 & $200.1 .2 . *$ & 200.1 .1 .4 & 53 & Accept \\
\hline 21 & 200.1 .2 .* & 200.1 .1 .5 & 22 & Accept \\
\hline 22 & $200.1 .2 . *$ & 200.1 .1 .5 & 3306 & Accept \\
\hline 23 & 200.1 .2 .* & 200.1 .1 .* & * & Deny \\
\hline 24 & $200.1 .2 .^{\star}$ & * & * & Accept \\
\hline 25 & * & 200.1 .1 .2 & 80 & Accept \\
\hline 26 & * & 200.1 .1 .2 & 443 & Accept \\
\hline 27 & * & 200.1 .1 .3 & 25 & Accept \\
\hline 28 & * & 200.1 .1 .4 & 53 & Accept \\
\hline 29 & * & * & * & Deny \\
\hline
\end{tabular}

Field Programmable Gate Array (FPGA). Whereas, research of the third type focuses on filtering mechanisms of firewalls, for instance, converting firewall rules into a tree structure which can process packets faster than a traditional sequential rule list.

In this section, we first conduct a review on the recent advances in the afore-discussed research focuses. Then, we present the achievements from our previous studies on Tree-rule firewall. These achievements are the underlying infrastructure of the new hybrid firewall proposed in this paper.

\subsection{Enhancing processing speed via rule conflict elimination}

Rule conflicts have come into focus of many researches on traditional firewalls. These firewalls use their listed rules to filter packets. The listed rules shown in Table 1, for example, illustrate how to regulate traffic traversing over the network presented in Fig. 1 in compliance with the network topology

In the context of firewall, rule conflicts can be classified into two categories, the ones causing speed issues and the ones causing security problems, respectively. As discussed in [2], [4] and [7], these rule conflicts result from shadowed rules and redundant rules, and they present critical impact on the performance of traditional firewalls.

Specifically, shadowed rules result in security problems on a traditional firewall. Rules blocking attack packets can be shadowed by some other rules with higher priorities (i.e., positioned ahead of them) and may not be used by the firewall at all. This, consequently, causes security problems and weakens the firewall [4]. Redundant rules decrease the processing speed of a firewall [2][4]. This is because they are redundant to other rules and waste the firewall's time to process them. Therefore, 
shadowed rules and redundant rules should be cleaned from a firewall rule set to improve the functional speed of a firewall.

To detect these rule conflicts, Al-Shaer and Hamed applied the set theory in their work published in [2]. Their approach is to map the original listed rules to a 'policy tree'. The conflicting rules and the types of the conflicts are reported after detection is completed. The authors further extended their methods to discover anomalies inside distributed networks [8].

The methods proposed in [7] also aim to discover rule conflicts. However, the proposed method in [7] is based on relational algebra techniques. It can discover more rule conflicts in comparison with the method suggested in [2]. The findings highlighted in [2], [7] and [8] suggest potential solutions to remove these problematic rules from a firewall rule set.

In addition, tools such as Binary Decision Diagrams (BDDs) [9], Constraint Logic Programming (CLP) [10] and Fireman Toolkit [11] were proposed to help analyze and remove rule conflicts from the rule set of a listed-rule firewall.

Although these studies [2][7][8][9][10][11] have introduced several schemes to deal with rule conflicts, their solutions are not satisfactory to this problem yet because listed rules are still in favor of all these proposed schemes.

\subsection{Enhancing processing speed via hardware im- plementation}

Fong et al. [12] implemented their firewall on FPGA devices to achieve a Terabit per second throughput for large and complex rule sets. They presented a scalable parallel architecture, named ParaSplit, for highperformance packet classification. Moreover, a rule set partitioning algorithm based on range-point conversion was proposed to reduce the overall memory requirement [12].

Likewise, Erdem and Carus [13] proposed a multipipelined and memory-efficient firewall to classify packets. They designed high throughput SRAM-based parallel and pipelined architectures on FPGAs. Hager et al. [14] proposed the Massively Parallel Firewall Circuits (MPFC) to generate customized firewall circuits in the form of synthesizable VHDL code for FPGA configuration. They claimed that MPFC circuits were highly parallel and could achieve a deterministic throughput of one packet per clock cycle.

However, the high speed performance achieved by the above-mentioned firewalls [12][13][14] was relied on special hardware (i.e., the FPGA) rather than on the design of a rule set architecture or development of a filtering algorithm.

2.3 Enhancing processing speed via advanced filtering mechanisms

$\mathrm{Ni}$ et al. [15] applied statistical analysis on two Transport layer protocol header fields of packets (i.e.,
Protocol and IP Address) based on the extracted features and the characteristics of multi-tree and dual-index strategy to decrease the firewall preprocessing time. This research used the 'data storage structure and search diagram' to filter packets. This structure is considered as a tree structure. However, the tree consists of only the fields of Protocol and IP address. It has no Port and Action fields in their tree. Moreover, firewall administrators still create firewall rules in a form of listed rule. Their approach compares the performance of their algorithm with Stochastic Distribution Multibit-trie (SDMTrie) algorithm [16] only. They claimed that their scheme was better than traditional firewalls and firewalls working with the SDMTrie algorithm. However, performance comparison with standard firewalls (e.g., IPTABLES, Cisco ACL) and any well known firewall algorithm is not presented.

Trabelsi et al. [17] proposed an analytical dynamic multilevel early packet filtering mechanism to enhance firewall performance. The proposed mechanism uses statistical splay tree filters that utilize traffic characteristics to minimize packet filtering time. The statistical splay tree filters are reordered according to the network traffic divergence upon certain threshold qualification (ChiSquare Test). They claimed that this method was faster than traditional methods because unwanted packets were rejected as early as possible, and the proposed mechanism could also be considered as a device protection mechanism against Denial-of-Service (DoS) attacks.

Hung et al. used B-Tree [18] to improve the speed of classifying and processing packets on firewall. They proposed a new two-dimensional early packet rejection technique based on the B-Tree. They defined a core firewall process as the 'Original Filter', and created their new scheme called 'Early rejected filter'. Their work focused on preventing unwanted packets and applied the 'Original Filter' to minimize packets traversing to the core firewall process. Their scheme can reduce firewall processing time under DoS attacks. However, under normal network operations (without DoS attack), their 'Early rejected filter' scheme may slightly increase firewall processing time.

Liu and Gouda [19] proposed 'Diverse Firewall Design' using tree-structured rules, which are converted from a rule list, to discover and eradicate rule conflicts. However, their work was still based on listed rules of traditional firewalls.

Zhao et al. [21] proposed to use 'goto' function inside listed-rule firewalls (e.g., a 'jump' command in IPTABLES). Although their rule structure looks like a tree structure, their sub-rules (or nodes) contain listed rules. Therefore, their firewalls are still deemed as Listed-rule firewalls and are time consuming when performing linear and sequential rule searching.

Likewise, although the methods proposed in [2][8] can convert firewall rules to a 'policy tree', the 'policy tree' cannot be considered as a tree-based filtering firewall mentioned in this paper. This is because the 'policy tree' is used only for rule conflicts discovery but not for filtering packets.

Apart from the afore-discussed three types of approaches, recent research has been investigating to devel- 
op a new generation firewall based on Software Defined Networking (SDN). For example, the firewalls proposed in [22], [23], [24] and [25] employ SDN and support centralized management like SDN switches and SDN router do. However, this SDN-based approach focuses on connectivity and compatibility with other SDN devices instead of firewall rule optimization.

\subsection{Background of Tree-rule firewall}

Chomsiri et al. have further studied firewall rules' problems, and published their interesting findings in [3] and [4]. They proposed a Tree-rule firewall to overcome these problems. The Tree-rule firewall not only organizes firewall rules in a tree structure as shown in Fig. 2 but also filters out unwanted packets in accordance with treestructured rules. To inspect a packet, the Tree-rule firewall first reads the relevant header fields from the packet. Then, the value of the first header field is compared with a firewall sub-rule stored in the root node of the tree. Afterwards, the firewall checks the other header fields sequentially against their respective tree nodes at the corresponding levels. Finally, a consequent action, such as an approval or a denial of access to the network, is taken on the packet. As shown in Fig. 2, packet header fields including Destination IP address (Dest IP), Destination Port (Dest Port), and Source IP address (Source IP) are taken into account in the example Tree rule. This tree structure eases the design of firewall rules and makes sure that they are conflict free, namely non-shadowed and nonredundant rules.

To further improve the processing speed of the Treerule firewall [4], we have proposed a stateful mechanism in [5]. However, this mechanism requires hashing calculation [6] at least once per packet. Therefore, the speed of

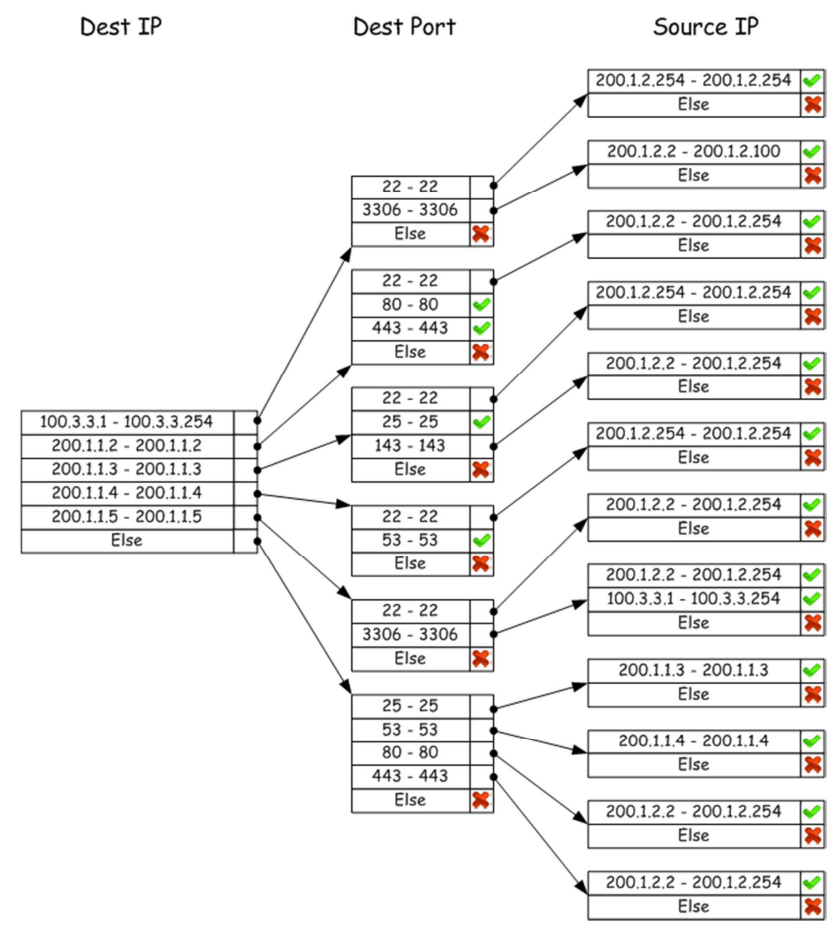

Fig. 2. A Tree rule structure created for an example network in Fig. 1. the firewall can be significantly improved if this complex hashing is eliminated. To achieve better speed performance, we propose a new hybrid firewall in this paper. The details of the proposed firewall are presented in Section 3.

\section{OUR APPROACH}

In this section, we propose a hybrid firewall which is a combination of a Tree-rule firewall and a traditional firewall. A Tree-rule firewall's GUI presented in our previous work [4] is used in the configuration phase to create tree rules, which are then converted to traditional conflict-free listed rules. During decision making, an incoming packet is verified against the listed rules sequentially until a match is found. Unlike the traditional firewalls, our hybrid firewall periodically re-arranges a sequence of rules. Each rule is independently moved to its suitable position in accordance with the number of matches with the incoming packets. For example, the rule matching with most packets is moved up to the top of the list in order to optimize the processing speed of the hybrid firewall.

\subsection{Methodology}

As shown in Fig. 3, there are four steps involved in the

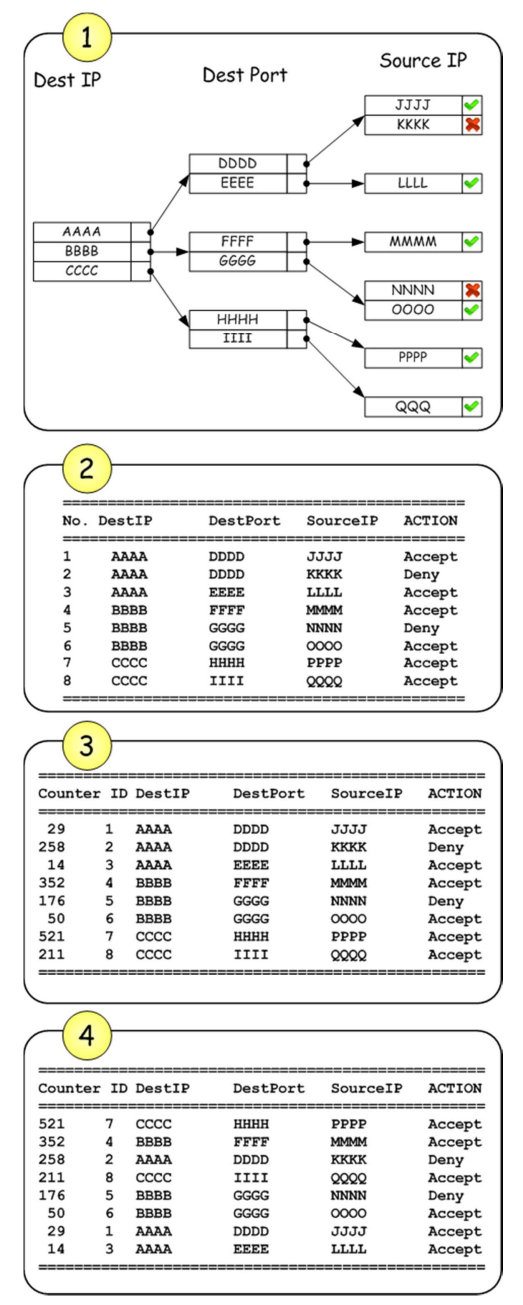

Fig. 3. Four steps of proposed scheme. 
process of our hybrid approach. In the first step shown in Fig. 3-(1), a tree rule is created using the GUI by a firewall rule designer. The created tree rule is then converted into listed rules as shown in Fig. 3-(2). The listed rule is then used in a core firewall for verifying against the header fields of an incoming packet. 'Counter' field shown in Fig. 3-(3) records the number of packets matched with each rule and is initially set to 0 for each rule. The 'Counter' of a rule will increase by one when a match between an incoming packet and the rule is confirmed. The counter determines which rule is most frequently matched. To reduce the computational time, the most frequently matched rule is relocated in the top of the list as shown in Fig. 3-(4). The counters of all the rules will be reset to 0 when a pre-determined 'Time interval' (e.g., 3 seconds) is reached. The 'Time interval' is specified by a firewall administrator.

When putting into practice, a range of IP addresses and a range of ports are applied in each line within nodes. The root node shown on the left-hand side of Fig. 2 consists of six lines. The range of numbers in each line does not overlap with the ranges of numbers in other lines within a same node. For example, the range [100.3.3.1100.3.3.254] does not overlap with the range [200.1.1.2200.1.1.2]. Likewise, the ranges of numbers in lines within a node (e.g., the first node of 'Dest Port' column) do not overlap with each other as well. These non-overlapping ranges allow us to transform a tree rule into a set of conflict-free listed rules.

Transforming a tree rule into a listed rule can be done for one rule path at a time. For example, the first rule path

$$
\begin{gathered}
([100.3 .3 .1-100.3 .3 .254]-->[22-22]-->[200.1 .2 .254 \\
\text { 200.1.2.254]-->Accept })
\end{gathered}
$$

can be transformed into the listed rule shown in Table 2 . The second rule path

$$
\text { ([100.3.3.1-100.3.3.254]-->[22-22]-->[Else]-->Deny) }
$$

can be transformed into the listed rule shown in Table 3.

TABLE 2

EXAMPLE OF A LISTED RULE TRANSFORMED FROM A RULE PATH

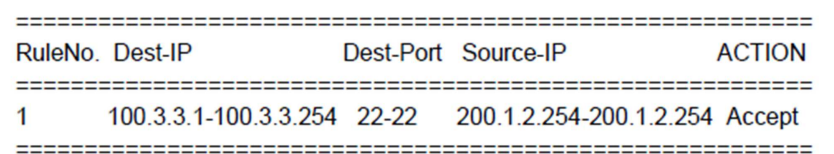

TABLE 3

EXAMPLE OF TWO LISTED RULES TRANSFORMED FROM A RULE PATH

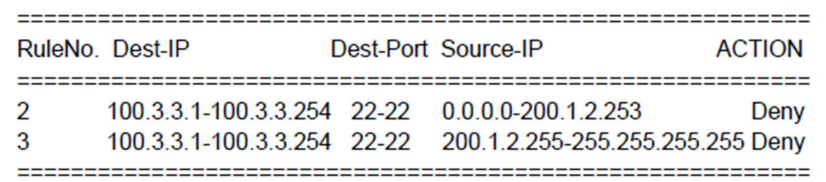

Bearing the same idea in mind, the tree rules shown in Fig. 2 can be transformed to the listed rules shown in Table 4 .
TABLE 4

THE LISTED RULES TRANSFORMED FROM THE TREE RULES IN FIG. 2

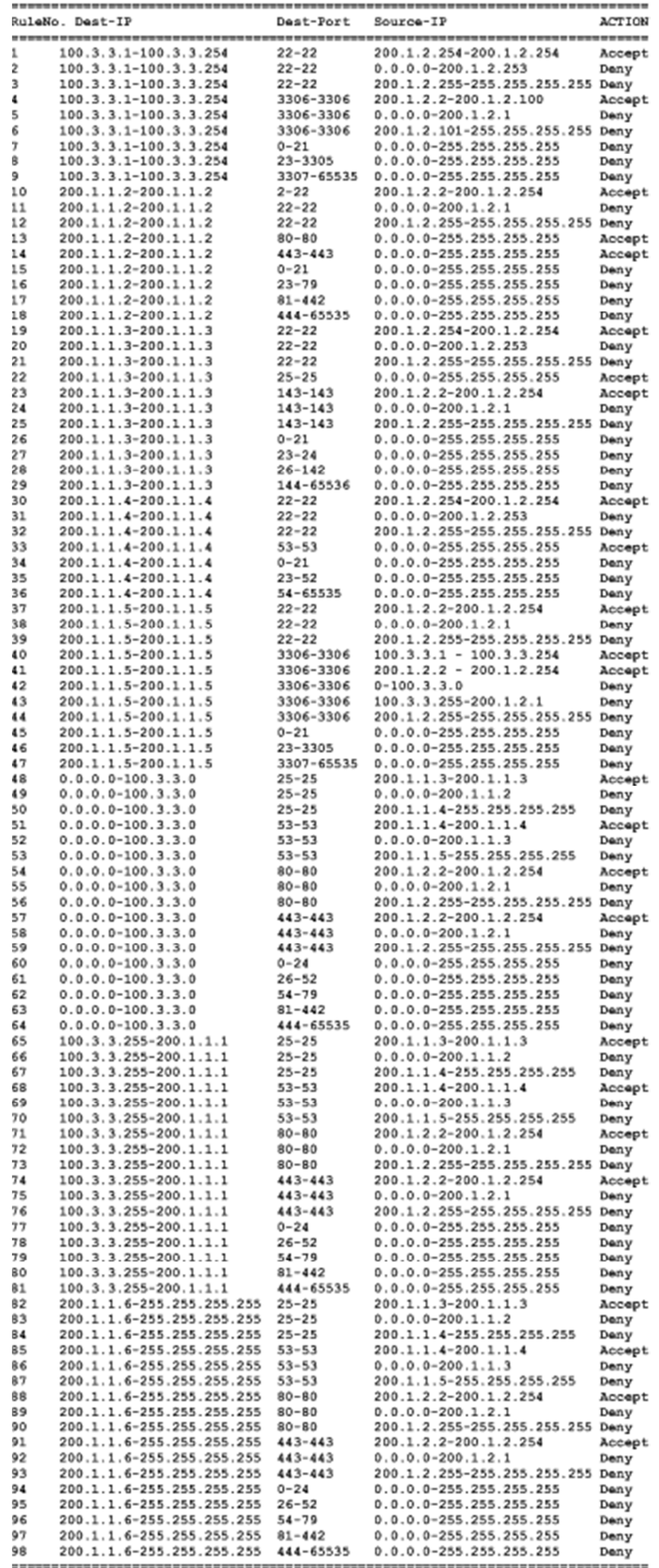

After designing and transforming tree rules into listed rules using the GUI, the listed rules shown in Table 4 are loaded into the memory of the core firewall for verifying 
against incoming packets. The counter of each rule will be increased individually when a packet is matched with a rule. All rules are sorted in descending order according to the value of a counter.

\subsection{Discussion on efficiency}

Although various methods [2][7][8][10][11][19] have been designed to minimize rule conflicts through rearrangement of those frequent matched rules to the top positions in a rule list, they do not guarantee that a conflict-free rule list can be reached.

Let us take the rule list illustrated in Table 1 as an example. When the network is under attack of worms, the last rule will be the most frequently matched rule within the list and is applied to drop those attack packets. Therefore, the last rule, namely Rule-29, will be re-positioned to the top of the list. This creates an undesirable consequence that all following incoming packets are blocked by Rule-29 even though they may be allowed by the other rules below. In contrast, individual listed rules created by our proposed scheme as shown in Table 4 can be moved to any position independently.

Moreover, given that the most frequently matched rules are listed at the bottom of a rule list, data transmission overhead of the aforementioned firewalls increase along with the expansion of their rule lists. This is because that it takes the firewalls' time to process unmatched rules before reaching the matched one and allowing/denying packets to pass through. According to our studies, 1000 redundant rules can reduce data transmission speed by approximately $10 \%$. The drop of speed

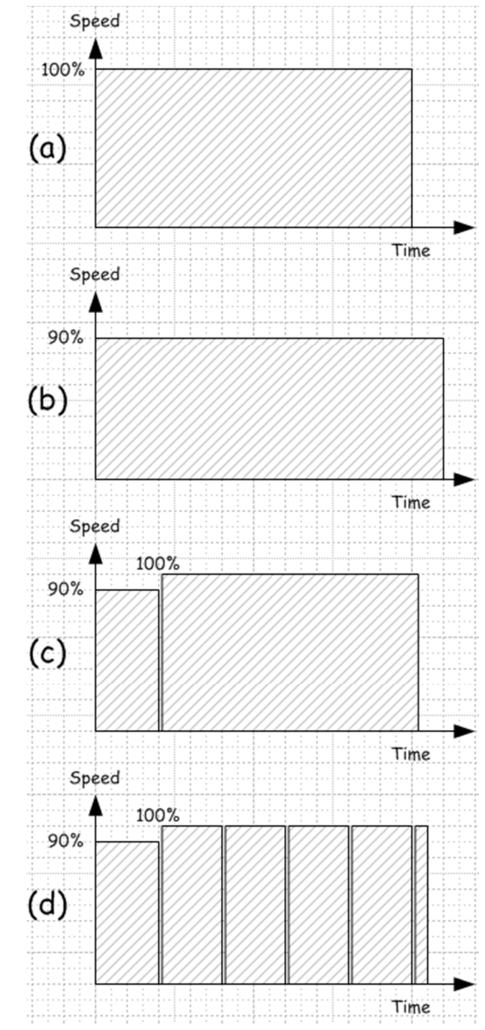

Fig. 4. Transmission speed versus transmission time depends on several factors, i.e., type of firewall [20] and CPU speed of the machine running the firewall.

The decrease of data transmission speed prolongs data transmission time of a system (e.g., time consumption for downloading the data increases $10 \%$ if data transmission speed drops by $10 \%$ as shown in Figs. 4 -(a) and (b) respectively). Moving the matched rule from the bottom of firewall rule list to the top position (e.g., from rule number 1000 to rule number 1 enhances the data transmission speed and shortens transmission time as illustrated in Fig. 4-(c). Using our proposed scheme, rule sorting is executed periodically for each specified time interval, such as 1 second, 3 seconds or 5 seconds. Sorting the firewall rules takes less time in comparison with rule matching. Time consumption for data transmission using our proposed scheme can be found in Fig. 4-(d). The time consumption shown in Fig. 4-(d) is more than that revealed in Fig. 4-(c) but less than that revealed in Fig. 4-(b).

In summary, there are five main factors determining time consumption, $T$, for data transmission and they are shown as follows.

- Time interval $(w)$

- Data size $(F)$

- Network speed $(S)$ (e)

- Efficiency of transmission speed before rule sorting

- Time for sorting rules $(g)$

Fig. 5 illustrates the time (T) used for transmitting data and the five main factors. $x$ axis and $y$ axis denote transmission time and transmission speed respectively. The figure reveals the relation between time $T$ used for data transmission and the five important factors (i.e., $w, F, S, e$ and $g$ ). In this example, we assume that the matched rule is at the bottom position of a rule list. The size of the rule list is 1000 , which decreases transmission speed by roughly $10 \%$ of the maximum speed. In the first state, transmission speed begins with $90 \%(e=0.9)$ until the time reach the Time Interval $(w)$. Then, the firewall takes time $g$ to sort its rules. We assume that the transmission speed during this period of time is 0 because the firewall is sorting

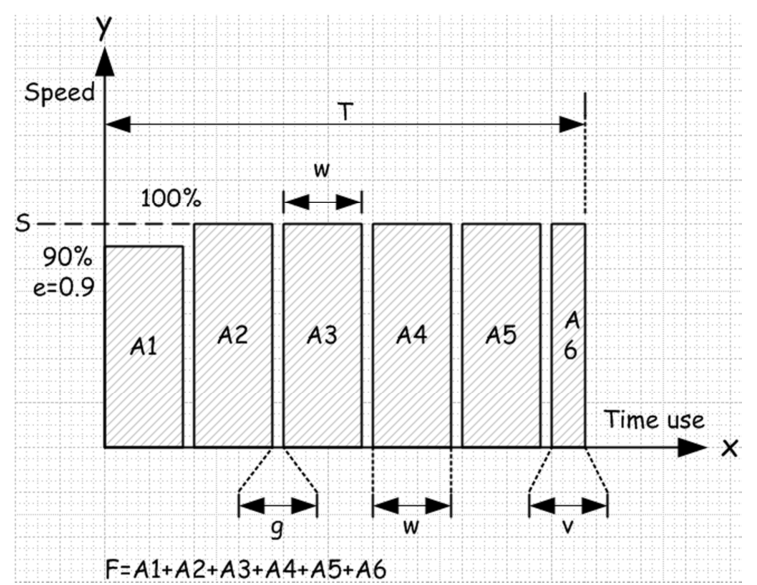

Fig. 5. Time $(T)$ used for data transmission and the five main factors $(w, F, S$, e and $g)$. 
its rules and not processing any packets. At this moment, data which have been transmitted is denoted as A1. After the rule sorting is complete, the firewall continues to process packets with its sorted rules. The transmission speed can peak at $100 \%$ because the matched rule has been moved up to the top position. When time interval $w$ ends, the firewall takes time $g$ to re-sort its rules again. This process repeats until the transmission of the last block of data (A6) is complete. The time $v$ used to transmit the last block may be smaller than $w$. The total amount of data $(F)$ transmitted is $F=A 1+A 2+A 3+A 4+A 5+A 6$.

The efficiency, $e$, is determined by the number of rules. We have created a special program to measure $e$ with 1000 rules on a $2.8 \mathrm{GHz} \mathrm{CPU}$ computer and $345 \mathrm{Mbps}$ network speed. We found that $e$ was approximately 0.9 . However, the value of $e$ may vary in different environments because it is influenced by multiple factors. Like $e$, $g$ is also determined by the number of rules. However, it equals to the base 2 logarithm of the number of rules because the Quick Sort [26] is used for rule sorting in this paper. Thus, $g$ increases slightly while the number of rules increases. We measured $g$ in the same environment where $e$ was done. We found that the value of $g$ was approximately 1 millisecond for 1000 rules. The $w$ is a free parameter and assigned by firewall administrators. It can be 1, 3 or 5 seconds. However, transmission time may be longer than usual if $w$ is specified inappropriately. The details of $w$ will be discussed later in Section 4 .

3.3 A mathematical model for measuring time consumption

Let

- $n$ denote the number of data blocks that do not include the first and the last data block (e.g., $n=4$ in Fig. 5),

- $F$ denote size of data being transmitted (in bits),

- $\quad e$ denote efficiency of transmission speed before sorting the rules, $0<e<1$,

- $S$ denote speed of network (in bits per seconds),

- $w$ denote time interval between two rule sortings (in seconds),

- $g$ denote time used for rules sorting (in seconds),

- $v$ denote the time span of transmitting the last block (in seconds), e.g., the time span of A6 in Fig. 5,

- $\quad u$ denote $v / w, 0<u<1$, and

- $\quad T$ denote the time used for data transmissions.

Then, we have

$$
\begin{aligned}
& F=e S w+n S w+S v \\
& F-e S w=n S w+S v \\
& (F-e S w) / S=n w+v=n w+u w \\
& (F-e S w) / S / w=n+u
\end{aligned}
$$

$$
\begin{aligned}
& n+u=(F-e S w) / S w \\
& n+u=(F / S w)-e
\end{aligned}
$$

The time $T$ used for data transmissions shown in Fig. 5 is defined as,

\section{$T$}

$$
\begin{aligned}
& =(w+g)+n(w+g)+v \\
& =(w+g)+n(w+g)+u w \\
& =(w+g)+n(w+g)+u(w+g)-u g \\
& =(w+g)+(n+u) \times(w+g)-u g
\end{aligned}
$$

Substituting Equation (1) into Equation (2), we have that

$T$

$$
\begin{aligned}
& =(w+g)+((F / S w)-e) \times(w+g)-u g \\
& =(w+g)-e(w+g)+\left(\frac{(w+g) F}{S w}\right)-u g \\
& =(1-e)(w+g)+\frac{F}{S}\left(1+\frac{g}{w}\right)-u g
\end{aligned}
$$

Equation (3) reveals that the larger the data size $F$ is, the longer time it takes a system to transmit data. Similarly, the higher the network speed $S$ is, the shorter time the system will take to transmit data. Moreover, $g, w$ and $e$ also play important roles in determining the time used for data transmission.

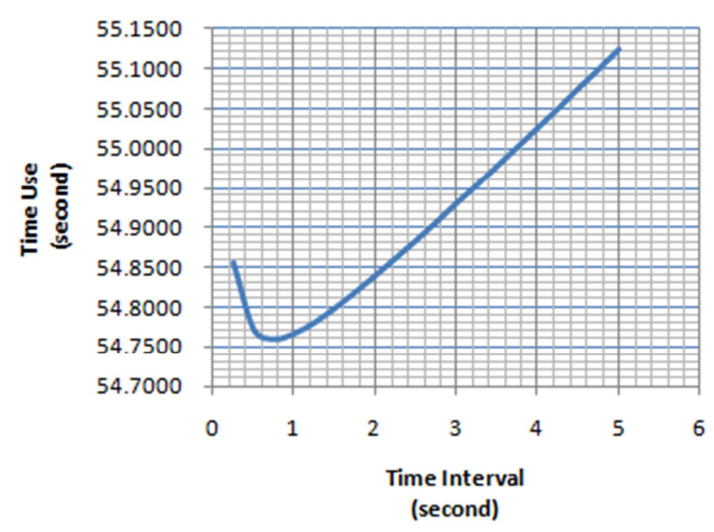

Fig. 6. Relation between Time use ( $T$ ) and Time Interval $(w)$

We have conducted a simple testing using this formula on Microsoft Excel, and given some input data for observing the result and output graphs. The results are shown in Fig. 6. We specified $F=2048 \mathrm{MB}$ (16384 Mbits), $S=300$ Mbps, $g=0.001$ seconds and $e=0.9$. We calculated consumption time $T$ for $w=0.25,0.50,0.75,1.00,1.25,1.50$, $1.75,2.00,2.25,2.50,2.75,3.00,3.25,3.50,3.75,4.00,4.25$, $4.50,4.75$ and 5.00 respectively. Fig. 6 shows a relation between the consumption time $T$ in the vertical axis and Time Interval $w$ in the horizontal axis. The curve of graph 
tells us that there is the optimal value of $w$ which can give the minimum consumption time $T$ for data transferring. In this case, $w=0.75$ causes $T=54.7603$, which is better than the values $T=54.7673, T=54.9313$ and $T=55.1243$ when $w=1.00,3.00$ and 5.00, respectively.

Regarding to the operation without using our proposed scheme, the firewall will take the time calculated using Equation (4) below for data transferring.

$$
T=\frac{F}{e S}
$$

Thus, in this example, without using our proposed scheme, the firewall will take time: $T=16384 /(0.9 * 300)=$ 60.6815 seconds. In contrast, using our proposed scheme, the transferring time can be saved for $9.76 \%$ for $w=0.75$, and $9.75 \%, 9.48 \%$ and $9.16 \%$ for $w=1,3$ and 5 seconds, respectively.

\subsection{Determining time interval $w$}

To determine the time interval $w$, we created a special program to measure a time used for sorting 1000 rules. We found that the sorting took less than 1 millisecond. Taking a four minute data transmission as an example, the sorting function is executed $80\left(=4^{*} 60 / 3\right)$ times if rules are sorted every 3 seconds. The overall time taken for rule sorting is merely 80 milliseconds which is very small in comparison with 4 minutes for the whole process. In the networks that have a small size of data transmission, setting the Time Interval to 3 seconds or 5 seconds may not be suitable because a time use $T$ of the firewall applying the proposed scheme may be bigger than a time use $T$ of the firewall without applying the proposed scheme (noting that the proposed scheme may waste firewall processing times due to the sorting time $g$ as shown in Equation (3)). Firewall administrators should calculate and set a good value of Time Interval $w$ to the firewall before using it. The proposed scheme focuses in cloud which mostly working with big size of data transferring. Thus, we can set the Time Interval $w$ to any value (e.g., 3 or 5 seconds) as long as the $T$ calculated from Equation (3) is less than the $T$ calculated from Equation (4).

We have found that the optimal Time Interval can be accurately estimated using Equation (5) below.

$$
w=\sqrt{\frac{F g}{S(1-e)}}
$$

We have derived Equation (5) based on Calculus from a function represented as $T=f(w)$, showing the relationship between the time use $(T)$ and the time interval $(w)$. The optimal $w$ occurs at the minimum point on the curve represented by this relation function (see Fig. 6) and can be obtained by differentiating $T$ with respect to $w$ as shown in Equation (6) below.

$$
\frac{d T}{d w}=0
$$

From Equation (3) in Section 3, ' $T$ ' can be calculated by:

$$
T=\frac{F}{S}\left(\frac{g}{w}+1\right)+(1-e)(w+g)-u g .
$$

Therefore, Equation (6) is equivalent to

$$
\begin{aligned}
& \frac{d}{d w}\left(\frac{F}{S}\left(\frac{g}{w}+1\right)+(1-e)(w+g)-u g\right) \\
= & \frac{d}{d w}\left(\frac{F}{S}\left(\frac{g}{w}+1\right)+(1-e)(w+g)\right) \\
= & \frac{d}{d w}\left(\frac{F g w^{-1}}{S}+\frac{F}{S}+(1-e) w+(1-e) g\right) \\
= & \frac{d}{d w}\left(\frac{F g w^{-1}}{S}+(1-e) w\right)=-\frac{F g}{S w^{2}}+(1-e)=0 .
\end{aligned}
$$

Thus, $w=\sqrt{\frac{F g}{S(1-e)}}$ that proves Equation (5).

In Fig. 6, we have calculated the time use $(T)$ for $w=0.25,0.50,0.75,1.00,1.25,1.50,1.75,2.00,2.25,2.50$, 2.75, 3.00, 3.25, 3.50, 3.75, 4.00, 4.25, 4.50, 4.75 and 5.00, respectively using Microsoft Excel. We have found that the optimal $w$ is 0.75 as we have discussed in subsection 3.2. With the same environments and parameters (e.g., the same value of $F, S, g$ and $e$ ), we have calculated $w$ using Equation (5), and found that the optimal $w$, which is 0.739008 . Therefore, it can be concluded that the optimal $w$ can be estimated by either of the two methods as follows.

- Using the Equation (3) to find the minimum $T$ for various input values of $w$

- Directly using Equation (5)

\section{IMPLEMENTATION AND EXPERIMENTATION}

Similar to our previous schemes [4][5], we implement the proposed schemes based on the Netfilter module [27][28][29]. We hook packets' events using a technique presented in [30] by calling the function named 'nf_register_hook' [30]. Before calling this function, the hooking function must be declared first, as such in the line: 'nfho.hook = hook_func'. When packets arrive at the firewall, the 'hook_func' will be called. It will receive several important parameters as shown below:

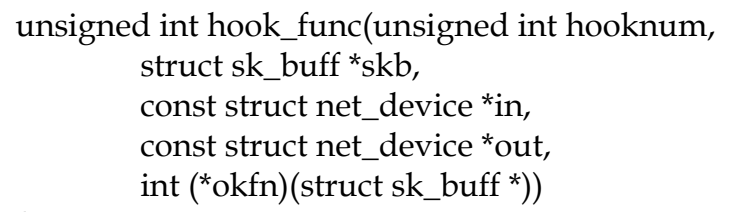




\subsection{Experimental setup and environment}

We create the Tree-rule firewall using C on Cent OS 6.3 Linux. It operates as a kernel module and runs in a kernel level. Our original firewall source code, 'firewall.c', is compiled into the 'firewall.ko' and can be executed by the command '\# insmod firewall.ko'. We develop rule editor GUI using C\# on Windows. The firewall rule is created by GUI and is sent to the core firewall running on Linux. The rule structure is modified for handling listed rules and counters information.

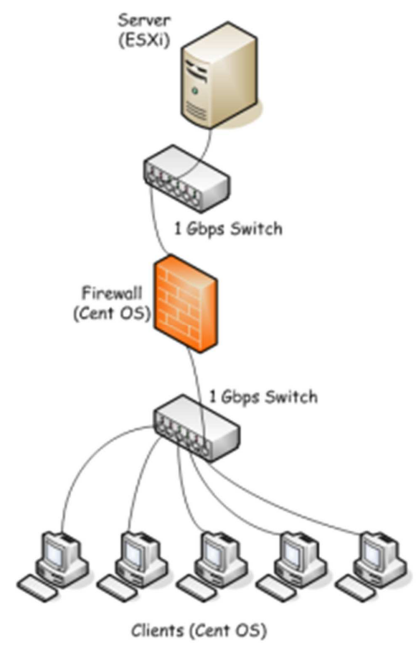

Fig. 7. Experiment with ESXi.

We evaluate the firewall on one Giga bits per second link speed LAN with seven standard PCs as shown in Fig. 7. The five clients and the firewall machine in this testbed are equipped with a $2.4 \mathrm{GHz}$ CPU and 4 GB RAM as well as a Cent OS 6.3. The server is equipped with a $2.8 \mathrm{GHz}$ CPU and 8 GB RAM as well as an ESXi (by VM Ware company) as OS/Hypervisor in a cloud environments. Within the server, we create five Virtual Machines (i.e., guest OSs) to serve as web servers (as shown in Fig. 8). Each Virtual Machine (VM) runs a Cent OS 6.3. All Ethernet links operate on 1 Gbps speed including network switches. Based on our experience, the performance on different hypervisors, such as VMW, ESXi, Microsoft Hy-

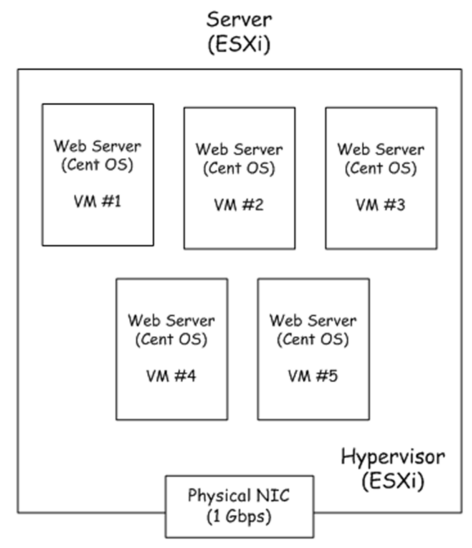

Fig. 8. Five Linux Web Servers within a ESXi Hypervisor.
per-V etc., are almost the same. Therefore, we decided to test on only on ESXi for the proposed work in this paper.

In our experimentation, time used for downloading big size of data (e.g., big files) is measured. To do so, we store a 4 GB file on VM \#1 (Server \#1), and 2 GB files on VM \#2 and VM \#3 respectively. We also place 1 GB files on VM \#4 and VM \#5, respectively. During evaluation, client \#1 downloads a file from VM \#1 only. Likewise, client \#i downloads a file from VM \#i only. We measure the downloading times on both 'automatic rule sorting' and 'non-automatic rule sorting' modes.

\subsection{Experiments}

The equation used in subsection 3.4 for finding optimal $w$ considers a single file containing firewall rules. However, in a real network, multiple files are simultaneously transmitted and each file may be matched with a different rule as well. Moreover, the size of each transmitting file may vary as well. Thus, finding the optimal "w" with multiple files is difficult. The selected $w$ of 3 makes administrators easy to manage the network and takes a little time for rule-sorting. For example, a computer LAB which is matched with one allowed rule, and open 3 hours for users to use it. Assume that $w$ is set to be 3 seconds on a firewall. In this case, the firewall will sort its rules $3 * 60 * 60 / 3=3,600$ times. If one round of rule sorting takes 0.002 seconds, the total sorting time will be $3600 * 0.002=7.2$ seconds, which is $0.067 \%$ in comparison to the 3 hours. This selected $w$ leads to a little sorting time in total. The firewall application developed using the proposed scheme can display information in its monitor screen to inform administrator which rules are the frequently matched rules. It is similar to the 'top' command in Linux which shows percentages of CPU used by each process. If we specify a too small $w$ (e.g., 0.5 or 1 seconds), it is hard
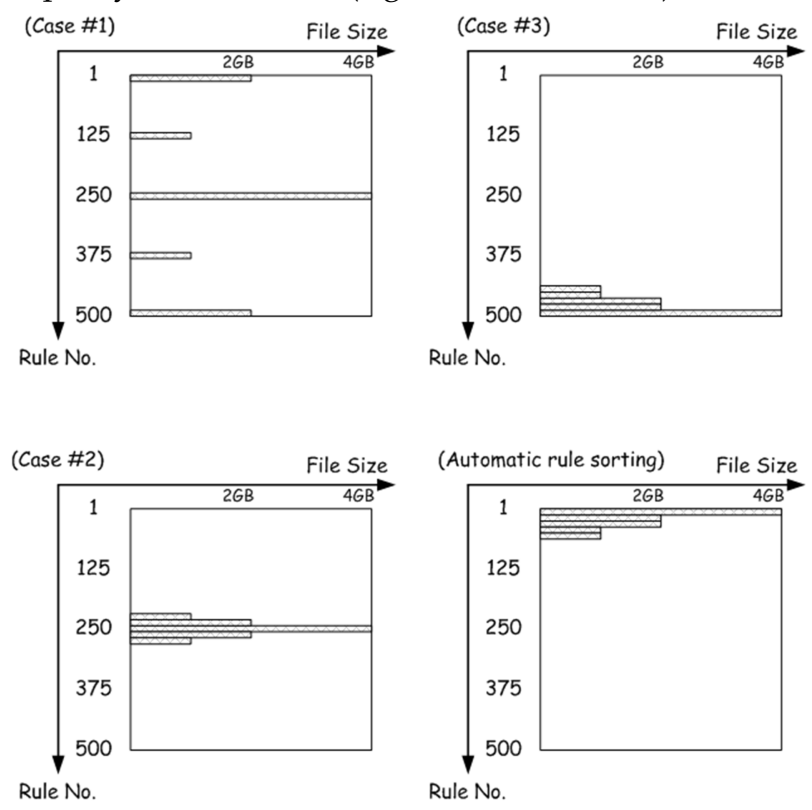

Fig. 9. Three cases of 'non automatic rule sorting' and a case of 'automatic rule sorting'. 

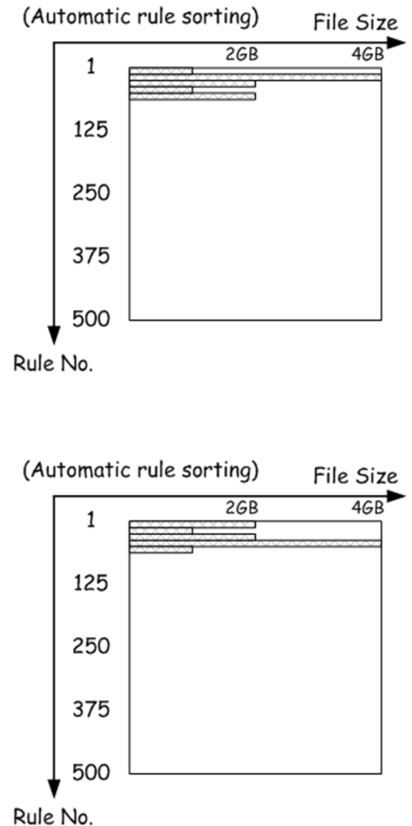

Fig. 10. Sequences of rules in 'automatic rule sorting'.

for administrators to read the information within such a short time window. In contrast, specifying a too big value of $w$ (e.g., 5 or 10 seconds) will result in slow reaction to apply administrators' preferences. Hence, the $w$ selected in our experiments is set to 3 seconds.

To begin with, we test on 3 cases with non-automatic rule sorting as shown in Cases \#1, \#2 and \#3 of Fig.9. We create 500 firewall rules and intentionally make rule \#250 match with the $4 \mathrm{~GB}$ file. In this case, the first rule and the last rule will match 2 GB files, while rules \#125 and \#375 match with 1 GB files. This is for measuring time consumption in average case.

Case \#2 is another average case for which five rules are in almost middle position. These files are matched with rules \#248, \#249, \#250, \#251 and \#252, respectively. In case \#3, we want to simulate the worst case by creating matched rules in positions 496, 497, 498, 499 and 500.

Secondly, we test with automatic rule sorting. We use a 3-second time interval (w), i.e., all rules are resorted every 3 seconds and a counter of each rule is reset to zero after all rules are resorted. Whilst five files are downloaded simultaneously, results of sorting may be different from the right bottom picture of Fig. 9. They may be sorted in many sequences as shown in Fig. 10.

Lastly, we test with 1000, 2000 and 4000 rules, respectively. Five files start to transfer at the same time. We start a timer at this point. All packets of files travel through the firewall rules. We stop the timer when the transfer of the last file is complete. In each case, we conduct the experimentation for five times, and the average result numbers are taken and highlighted in Table 5 .

Case \#1 and Case \#2 in Table 5 are average cases, whose results are very similar. Case \#3 is the worst case that takes a longer time in comparison with Case \#1 and Case \#2. In three cases, the downloading times are longer when the number of rule is increased. In the case of 'au- tomatic rule sorting', firewall rules are sorted every 3 seconds so that five rules matching with fives active connections are moved to the top five positions. In other words, these rules are moved to rules with numbers 1, 2, 3,4 and 5 . The firewall has to verify packets against only the first five rules and is not necessary to process the remaining unmatched rules. Consequently, time consumption in this case is the smallest in comparison with the other cases. Moreover, the time consumptions for 500, 1000,2000 , and 4000 rules are slightly different. The percentages of time saving are presented in Table 6. As shown in Table 6, our scheme can reduce the processing time of the firewall with 500 rules by $8.17 \%$ on average. More time is saved in the cases with bigger rule sizes. For example, the proposed method saves $60.89 \%$ of the time for the case with 4,000 rules as shown in Table 6 .

TABLE 5

TIME CONSUMPTION FOR TRANSFERRING FILES FROM SERVERS TO CLIENTS (MINUTES)

\begin{tabular}{|c|r|r|r|r|}
\hline \multirow{2}{*}{ Case } & \multicolumn{4}{|c|}{ Number of Rules } \\
\cline { 2 - 5 } & 500 & 1,000 & 2,000 & 4,000 \\
\hline$\# 1$ & 4.40 & 4.79 & 5.66 & 7.34 \\
$\# 2$ & 4.42 & 4.82 & 5.58 & 7.33 \\
$\# 3$ & 4.82 & 5.65 & 7.32 & 10.66 \\
Automatic rule sorting & 4.05 & 4.13 & 4.21 & 4.17 \\
\hline
\end{tabular}

TABLE 6

TIME SAVE IN PERCENTAGE

\begin{tabular}{|c|r|r|r|r|}
\hline \multirow{2}{*}{ Time Save (\%) } & \multicolumn{4}{|c|}{ Number of Rules } \\
\cline { 2 - 5 } & 500 & 1,000 & 2,000 & 4,000 \\
\hline avg of case \#1 and \#2 & 8.17 & 14.06 & 25.10 & 43.16 \\
case \#3 & 15.99 & 26.91 & 42.50 & 60.89 \\
\hline
\end{tabular}

Apart from testing on ESXi Hypervisor, we also conduct experiments setting up a small LAN with four servers, four clients and our Tree-rule firewall in the perimeter. We compare the performance of our proposed firewall with IPTABLES, the most popular open-source firewall, using multiple sets of rule having different size. All computers including the firewall machine in this testbed are equipped with a $2.2 \mathrm{GHz}$ CPU and 8 GB RAM. The firewall's OS is Cent OS 6.3 while the Back Track 5 R3 was used as OS for servers and clients. The servers generate packets using 'hping3' command with '-flood' parameter to create and send the packets as fast as possible. This test uses 1440 bytes packet size. We choose a bigger packet size because HTTP typically uses packet size of 1400-1500 bytes.

The worst cases (when all packets are matched with the last rules) can be tested by creating one matched rule at the bottom position of firewall rule list. Apart from the last rule, other rules are considered unmatched rules. This condition is similar to case \#3 of the previous experimentation but using one matched rule at the bottom of rule list.

We measure speeds of IPTABLES with different rule size, e.g, 100, 250, 500, 1000, 1500, 2000, 2500, 3000, 3500 
and 4000 rules. The 'hping3' command with '-flood' can throttle the firewall to operate with its maximum speed (throughput). With no rule (rule size=0), IPTABLES can process 30956 packets per second, as shown in Table 7. In Tables 7, the firewall speed was represented in term of packets per second, and mega bytes per second. The data were calculated using 1440 bytes packet size.

\section{TABLE 7}

SPEED ACHIEVED THROUGH IPTABLES

\begin{tabular}{|r|r|r|r|}
\hline \multirow{2}{*}{ Number of rules } & \multicolumn{2}{|c|}{ Speed } & \multirow{2}{*}{ Drop (\%) } \\
\cline { 2 - 3 } & Packets/sec & Mega Bytes/sec & \\
\hline 100 & 30,956 & 42.51 & - \\
250 & 27,964 & 38.40 & 9.67 \\
500 & 25,099 & 34.47 & 18.92 \\
1,000 & 21,226 & 29.15 & 31.43 \\
1,500 & 16,202 & 22.25 & 47.66 \\
2,000 & 13,172 & 18.09 & 57.45 \\
2,500 & 11,103 & 15.25 & 64.13 \\
3,000 & 9,580 & 13.16 & 69.05 \\
3,500 & 8,471 & 11.63 & 72.64 \\
4,000 & 7,526 & 10.34 & 75.69 \\
& 6,653 & 9.14 & 78.51 \\
\hline
\end{tabular}

We can see, the speed of IPTABLES drops from 42.51 $\mathrm{MB} / \mathrm{s}$ to $22.25 \mathrm{MB} / \mathrm{s}(47.66 \%)$ having 1000 rules. The percentage of speed drop increases when the firewall processes a bigger rule size.

We also test the proposed firewall with the same condition (as we tested IPTABLES) by disabling the feature 'Automatic rule sorting'. As shown in Table 8, speed of our firewall operating with rule size $=20000,30000,40000$, $50000,60000,70000$ and 80000 indecate that our firewall operates faster than the IPTABLES approximately by 20 times. For rule size $=1000$, speed of our firewall drops only $7.43 \%$. In comparison, IPTABLES speed drops by $47.66 \%$. The two plots as shown through Figure 11 and Figure 12 translate corresponding data present in Table 7 and Table 8. In the two plota, vertical axis of the graph represents speeds of firewall in MByte/sec whereas the horizontal axis represents numbers of rules.

TABLE 8

SPEED OF PROPOSED FIREWALL WITHOUT 'AUTOMATIC RULE SORTING'

\begin{tabular}{|r|r|r|r|}
\hline \multirow{2}{*}{ Number of rules } & \multicolumn{2}{|c|}{ Speed } & \multirow{2}{*}{ Drop (\%) } \\
\cline { 2 - 3 } & Packets/sec & Mega Bytes/sec & \\
\hline 100 & 30,956 & 42.51 & - \\
250 & 30,475 & 41.85 & 1.55 \\
500 & 30,254 & 41.55 & 2.27 \\
1,000 & 29,755 & 40.86 & 3.88 \\
2,000 & 28,658 & 39.36 & 7.43 \\
5,000 & 27,251 & 37.42 & 11.97 \\
10,000 & 23,563 & 32.36 & 23.88 \\
20,000 & 19,288 & 26.49 & 37.69 \\
30,000 & 14,338 & 19.69 & 53.68 \\
40,000 & 11,602 & 15.93 & 62.52 \\
50,000 & 9,556 & 13.12 & 69.13 \\
60,000 & 8,384 & 11.51 & 72.92 \\
70,000 & 7,156 & 9.83 & 76.88 \\
80,000 & 6,556 & 9.00 & 78.82 \\
& 5,860 & 8.05 & 81.07 \\
\hline
\end{tabular}

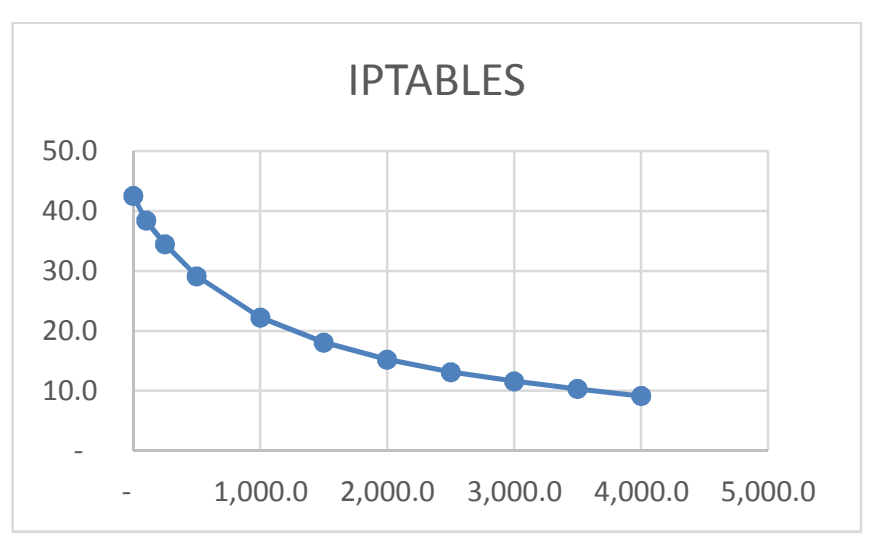

Fig. 11. Speed of IPTABLES (represented in graph)

\section{The Proposed Firewall}

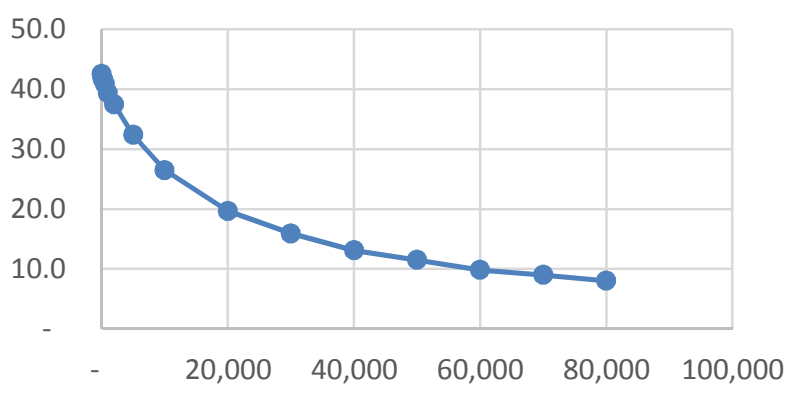

Fig. 12. Speed of Proposed Firewall without 'Automatic rule sorting' (represented in graph)

We perform more experiments for the proposed firewall to compare between operations with and without 'Automatic rule sorting'. Experimental results are presented in Table 9 and Figure 13.

TABLE 9

SPEED OF PROPOSED FIREWALL WITH 'AUTOMATIC RULE SORTING'

\begin{tabular}{|r|r|r|r|}
\hline \multirow{2}{*}{ Number of rules } & \multicolumn{2}{|c|}{ Speed } & \multirow{2}{*}{ Drop (\%) } \\
\cline { 2 - 3 } & Packets/sec & Mega Bytes/sec & \\
\hline 100 & 30,948 & 42.50 & - \\
250 & 30,672 & 42.12 & 0.92 \\
500 & 30,663 & 42.11 & 0.95 \\
1,000 & 30,412 & 41.76 & 1.76 \\
2,000 & 30,316 & 41.63 & 2.07 \\
5,000 & 30,060 & 41.28 & 2.90 \\
10,000 & 29,821 & 40.95 & 3.67 \\
20,000 & 29,487 & 40.49 & 4.75 \\
30,000 & 29,043 & 39.88 & 6.18 \\
40,000 & 28,488 & 39.12 & 7.97 \\
50,000 & 28,272 & 38.83 & 8.67 \\
60,000 & 27,696 & 38.03 & 10.53 \\
70,000 & 27,741 & 38.10 & 10.39 \\
80,000 & 27,444 & 37.69 & 11.34 \\
& 26,936 & 36.99 & 12.99 \\
\hline
\end{tabular}




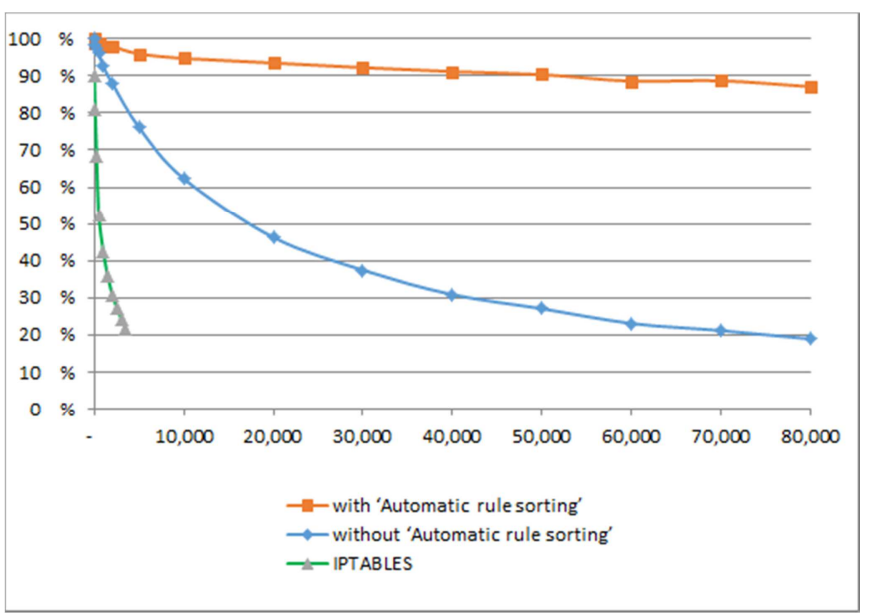

Fig. 13. Comparision of Firewalls' speeds

With rules size $=1000$ in Table 9, the proposed firewall with 'Automatic rule sorting' gives $2.07 \%$ of speed drop whereas operating without 'Automatic rule sorting' gives $7.43 \%$ (see Table 8 ). Figure 13 shows speed comparison for three firewalls, i.e., (1) the proposed firewall operating with 'Automatic rule sorting', (2) the proposed firewall operating without 'Automatic rule sorting', and (3) IPTABLES. The results shown through these graphs confirm that our proposed firewall with 'Automatic rule sorting' operates faster than IPTABLES significantly, and particulay with large size of rule set.

\section{CONCLUSION AND FUtURE WORKS}

In this paper, we have proposed a hybrid Tree-rule firewall which reduces processing time in verifying packets. The proposed firewall applies the concepts of Tree-rule firewall in designing conflict-free rules and the concepts of traditional firewall in decision making. Verifying incoming network packets against conflict-free listed rules contributes a more secure and faster processing firewall. Counters are introduced to analyze which rules match with the most packets. The rules are sorted according to the counters periodically, and the most frequently matched rules are moved to the top positions. As such, time spent in rule matching can be further reduced because a match can most possibly be found in the first few rules.

We have also proposed a mathematical model to illustrate a relation between 'time use' for data transferring and other relevant factors, especially 'time interval'. Moreover, we have proposed an equation for calculating an optimal 'time interval' with a mathematical proof based on Calculus.

Experiments have been conducted using our implemented testbed for evaluating the performance of our proposed hybrid firewall on a big size of data transferring. The experimental results show that our scheme can reduce firewall processing time significantly. For our future research, we will further improve and test the pro- posed firewall in other environments.

\section{REFERENCES}

[1] W. Cheswick, S. Bellovin, A. Rubin, Firewalls and Internet Security: repelling the wily hacker, Addison-Wesley Professional, 2003.

[2] E. Al-Shaer, H. Hamed, Firewall policy advisor for anomaly detection and rule editing, in: Proceedings of the IEEE/IFIP Integrated Management, IM, 2003, pp. 17-30.

[3] T. Chomsiri, X. He, P. Nanda, Limitation of listed-rule firewall and the design of Tree-rule firewall, in: Proceedings of the 5th International Conference on Internet and Distributed Computing Systems, China, 2012, pp. 275-287.

[4] X. He, T. Chomsiri, P. Nanda, Z. Tan, Improving cloud network security using the Tree-rule firewall, Future Generation Computer Systems, Elsevier, 30 (2014) 116-126.

[5] T. Chomsiri, X. He, P. Nanda, Z. Tan, A Stateful Mechanism for the Tree-rule Firewall, 2014 IEEE 13th International Conference on Trust, Security and Privacy in Computing and Communications (TrustCom. 2014), 2014, pp. 122-129.

[6] P. Ayuso, Netfilter's Connection Tracking System, LOGIN; , The USENIX magazine, 32 (2006) 34-39.

[7] C. Pornavalai. T. Chomsiri, Firewall Policy Analyzing by Relational Algebra, In: proceeding of the 2004 International Technical Conference on Circuits/Systems, Computers and Communications (ITC-CSCC), 2004, pp. 214-219.

[8] E. Al-Shaer, H. Hamed, R. Boutaba, M. Hasan, Conflict classification and analysis of distributed firewall policies, IEEE Journal on Selected Areas in Communications 23 (10) (2005) 2069-2084.

[9] S. Hazelhusrt, Algorithms for Analyzing Firewall and Router Access Lists, Technical Report TR-WitsCS-1999, Department of Computer Science, University of the Witwatersrand, 1999.

[10] P. Eronen, J. Zitting, An Expert System for Analyzing Firewall Rules, In: Proceedings of the 6th Nordic Workshop on Secure IT-Systems (NordSec), 2001, pp. 100-107.

[11] L. Yuan, J. Mai, Z. Su, FIREMAN: A toolkit for Firewall modeling and analysis, In: Proceedings of the 2006 IEEE Symposium on Security and Privacy, 2006, pp. 199-213.

[12] Fong, Jeffrey, Xiang Wang, Yaxuan Qi, Jun Li, and Weirong Jiang. "ParaSplit: a scalable architecture on FPGA for terabit packet classification." In High-Performance Interconnects (HOTI), 2012 IEEE 20th Annual Symposium on, pp. 1-8. IEEE, 2012.

[13] Erdem, Oğuzhan, and AydinCarus. "Multi-pipelined and memory-efficient packet classification engines on FPGAs." Computer Communications (2015).

[14] Hager, Sven, Frank Winkler, Bjorn Scheuermann, and Klaus Reinhardt. "MPFC: Massively Parallel Firewall Circuits." In Local Computer Networks (LCN), 2014 IEEE 39th Conference on, pp. 305-313. IEEE, 2014.

[15] Ni, Cuixia, Guang Jin, and Xianliang Jiang. "A New Multi-tree and Dual Index based Firewall Optimization Algorithm." TELKOMNIKA Indonesian Journal of Electrical Engineering 11, no. 5 (2013): 2387-2393.

[16] Fengjun S, Yingjun P, Xuezeng P, Bin B. Research on a Stochastic Distribution MultibitTrie Tree IP Classification Algorithm. Journal of Communications (in Chinese). 2008; 29(7): 109-117.

[17] Trabelsi, Zouheir, Mohammad M. Masud, and KilaniGhoudi. "Statistical dynamic splay tree filters towards multilevel firewall packet filtering enhancement." Computers \& Security 53 (2015): 109-131.

[18] Hung, Nguyen Manh, and Vu Duy Nhat. "B-tree based two- 
dimensional early packet rejection technique against DoS traffic targeting firewall default security rule." In Computational Intelligence for Security and Defense Applications (CISDA), 2014 Seventh IEEE Symposium on, pp. 1-6. IEEE, 2014.

[19] A. Liu, M. Gouda, Diverse Firewall Design, IEEE transaction on parallel and distributed systems 19 (9) (2008) 1237-1251.

[20] "1000 redundant rules of IPTABLES (TCCSI-2015-01-0032.R1)", https://www.youtube.com/results?search_query=TCCSI2015-01-0032.R1, 2016

[21] L. Zhao, A. Shimae, H. Nagamochi, Linear-tree rule structure for firewall optimization, In: Proceedings of Communications Internet and Information Technology, 2007, pp. 67-72.

[22] M. Kang, J. Choi, H. Kwak, I. Kang, M. Shin, J. Yi, Formal modeling and verification for SDN firewall application using pACSR, In Electronics, Communications and Networks IV: Proceedings of the 4th International Conference on Electronics, Communications and Networks (CECNET IV), Beijing, China, 2014, pp. 155.

[23] S. Kumar, R. Perumalraja, Establishing User-Defined Firewall in Software Defined Network, International Journal of Research 2(6)(2015), pp. 28-31.

[24] M. Suh, S. Park, B. Lee, S. Yang, Building firewall over the software-defined network controller, In Advanced Communication Technology (ICACT), 2014 16th International Conference, 2014, pp. 744-748.

[25] H. Hu, G. Ahn, W. Han, Z. Zhao, Towards a reliable sdn firewall, Presented as part of the Open Networking Summit (ONS 2014), 2014.

[26] "Java Applets Centre - University of Canterbury", http://www.cosc.canterbury.ac.nz/mukundan/dsal/QSort.ht $\mathrm{ml}, 2015$

[27] R. Rosen, Netfilter, Linux Kernel Networking, Apress, (2014) 247-278.

[28] The netfilter.org project, 2014, http://www.netfilter.org/.

[29] P. Ayuso, Netfilter's Connection Tracking System, LOGIN;; The USENIX magazine, 32 (2006) 34-39.

[30] Fidel, Vidal, and José María. "Mecanismopara el accesopúblico a servidores con direccionamientoprivado." (2011).

Thawatchai Chomsiri is a Ph.D. student at the Faculty of Engineering and Information Technology (FEIT) of the University of Technology, Sydney (UTS), Australia. He is also an Assistant Professor at the Department of Information and Communication Technology in the Faculty of Informatics of the Mahasarakham University, Thailand. Mr. Chomsiri has 17 years of experience in industry, teaching and research. His research interests are computer networking, and computer and network security.

Xiangjian He is a Professor of Computer Science. He is also the Director of Computer Vision and Recognition Laboratory and a coleader of the Network Security Research group at the University of Technology, Sydney (UTS). He is an IEEE Senior Member. He has been awarded Internationally Registered Technology Specialist by International Technology Institute (ITI). His research interests are network security, image processing, pattern recognition and computer vision.

Priyadarsi Nanda is a Senior Lecturer in the School of Computing and Communications, and is a Core Research Member at the Centre for Real-time Information Networks (CRIN) at the University of Technology, Sydney (UTS). His research interests are network QoS, network securities, assisted health care using sensor networks, and wireless networks. Dr Nanda has over 23 years of experience in teaching and research.
Zhiyuan Tan is a Postdoctoral Researcher in Services, Cyber security and Safety Research Group, Faculty of Electrical Engineering, Mathematics and Computer Science, University of Twente, Netherlands. His research interests are network security, pattern recognition, machine learning and distributed computing. 